\title{
APPROXIMATE REALIZATION OF VALVE DYNAMICS WITH TIME DELAY
}

\author{
Jan van Helvoirt ${ }^{*, * *, 1}$ Okko Bosgra ${ }^{*, * * *}$ \\ Bram de Jager* Maarten Steinbuch*
}

\author{
* Control Systems Technology Group, Mechanical \\ Engineering Department, Technische Universiteit \\ Eindhoven, P.O. Box 513, 5600 MB, Eindhoven, \\ The Netherlands \\ ** Flow \& Structural Dynamics Department, TNO TPD, \\ P.O. Box 155, 2600 AD, Delft, \\ The Netherlands \\ *** Delft University of Technology, Delft Center for \\ Systems and Control, Mekelweg 2, 2628 CD, Delft, \\ The Netherlands
}

\begin{abstract}
This paper describes the model identification of control valve dynamics with a time delay. An experimental set-up is used to perform both frequency response and step response measurements on the control valve. An approximate realization algorithm and a new time delay estimation method are applied on the experimental data. The results illustrate that the suggested approach provides a good time delay estimate and a reasonable state space description for the valve dynamics. Copyright (C)2005 IFAC
\end{abstract}

Keywords: approximate realization, identification, time delay, valve dynamics.

\section{INTRODUCTION}

In general, the minimal state space realization problem for linear time-invariant (LTI) systems refers to finding a state space description that explains some given data about an LTI system. The available data are typically the impulse response of the system, the step response, or more general input-output or frequency measurements. The formulation of the realization problem originates from research in the early 1960s on LTI system dynamics (Gilbert, 1963; Kalman, 1963). A survey of results that are related to the minimal state space realization problem is given in (Schutter, 2000).

1 Corresponding author is J. van Helvoirt, e-mail: j.v.helvoirt@tue.nl
Next to addressing a fundamental issue in system theory, the minimal realization problem is also related to the more involved problem of system identification, in particular to the field of subspace identification. Loosely speaking, system identification can be seen as a realization problem that is complicated by noise, nonlinear effects, modeling errors and so on. In this context, the method given in (Kung, 1978) can be regarded as a straightforward system identification method. The method results in an approximate realization for a measured impulse response by utilizing the algorithm from (Ho and Kalman, 1965) in combination with a singular value decomposition (SVD).

The advantage of the mentioned approximate realization method is its straightforward numerical implementation, the manifest relation with 
the original data throughout the algorithm, and its computational simplicity. However, one of the problems that is not explicitly taken into account by this method, is the presence of a time delay in the dynamical system.

Numerous strategies have been proposed to identify the time delay (dead time) in a dynamical system. In (Pintelon and van Biesen, 1990) a maximum likelihood estimator is proposed to estimate the transfer function of a linear continuous time system with time delay. A drawback of this method is the fact that a good initial estimate is required in order to guarantee convergence of the cost function to its global minimum. The method presented in (Jang et al., 2001) uses least squares estimation to determine model parameters from a time-domain residual. This approach requires an inverse Laplace transformation and knowledge of the model structure in advance. Adaptive identification schemes for systems with time delay are suggested in (Tuch et al., 1994; Orlov et al., 2002). These methods are claimed to perform well but requirements like measurable states and/or oscillating inputs limit their practical value.

This paper presents the application of an approximate realization algorithm in combination with a novel time delay estimation method. The dynamical system under study is a control valve that is used as an actuator in an industrial compressor test rig. The motivation to analyse the dynamic behavior of this control valve, comes from the research that is being carried out with the mentioned compression system (Helvoirt et al., 2004). Next to this practical reason, the control valve provides a test bed for evaluating the approximate realization algorithm and the time delay estimation method as identification tools.

The paper is organized as follows. Section 2 introduces the approximate realization method and the related time delay estimation method. Furthermore, the experimental set-up for the control valve is discussed. Section 3 starts with an overview of various measurement results from the control valve set-up. Then the identification of the control valve dynamics, by means of the approximate realization algorithm and time delay estimation method, are addressed and the obtained results are presented. Finally, the main results are summarized and discussed in Section 4.

\section{PRELIMINARIES}

In this section the used theoretical and numerical concepts are presented, as well as the experimental set-up that was used. Firstly, an algorithm that calculates an approximate realization from impulse response data, is discussed. Then, the time delay estimation method is introduced that was used to obtain an estimate of the delay in the system under study. Finally, some details of the experimental set-up for the investigated control valve are given.

\subsection{Approximate realization with time delay}

The term realization refers to a state-space representation of a given input-output mapping. When the input-output data set is of finite dimensions or when the data are corrupted with noise we speak of a partial or approximate realization.

A straightforward way to obtain an approximate realization for a discrete-time LTI system, is via the construction of a Hankel matrix from a finite sequence of measured Markov parameters. Note that for a discrete-time LTI system the Markov parameters represent the impulse response of the system. An approximate realization of appropriate order $\rho$, is then calculated from the singular value decomposition (SVD) of this Hankel matrix (Kung, 1978). The algorithm reads ${ }^{2}$

(1) Construct the Hankel matrix $T$ according to

$$
T_{i, j}= \begin{cases}G_{i+j-1} & \text { for } i+j \leq n+1 \\ 0 & \text { for } i+j>n+1\end{cases}
$$

from the step response sequence $\left\{S_{k}\right\}_{k=0}^{n}$

(2) Compute the SVD $H_{e}=U \Sigma V^{T}$ of the matrix $H_{e}=T(1: r, 1: r)$ with $r=\lfloor n / 2\rfloor$

(3) Construct the matrices $U_{\rho}=U(:, 1: \rho)$, $V_{\rho}=V(:, 1: \rho)$, and $\Sigma_{\rho}=\Sigma(1: \rho, 1: \rho)$

(4) Construct the matrices

$$
\begin{aligned}
& H_{a}=T(2: \rho+1,1: \rho), H_{b}=T(1: \rho, 1), \\
& H_{c}=T(1,1: \rho), \text { and } H_{d}=S_{0}
\end{aligned}
$$

(5) Construct the system matrices of the realization

$$
\begin{aligned}
& A=\Sigma_{\rho}^{-\frac{1}{2}} U_{\rho}^{T} H_{a} V_{\rho}^{T} \Sigma_{\rho}^{-\frac{1}{2}} \\
& B=\Sigma_{\rho}^{-\frac{1}{2}} U_{\rho}^{T} H_{b} \\
& C=H_{c} V_{\rho}^{T} \Sigma_{\rho}^{-\frac{1}{2}} \\
& D=H_{d}
\end{aligned}
$$

The selection of a value for the order $\rho$ of the realization determines the singular values that are considered to be contributing to the order of the approximate realization. Note that when $\rho$ is taken too large, the noise present in the Markov parameters is modelled as a part of the realization.

\footnotetext{
2 The used Matlab-like notation (:) is a shorthand for an entire row or column of a matrix. Similarly, $(a: b)$ indicates consecutive rows or columns.
} 
Now consider a discrete time LTI system with a time delay of $d$ samples. In the time domain a delay of $d$ samples results in, for example, a step response $y(k)$ where the first $d$ samples are equal to zero. The time delay enters the numerator of the transfer function as $z^{-d}$ and hence it results in a pole at $z=0$ with multiplicity $d$. It can be shown that an approximate realization for systems with a time delay, often fails to describe the dynamics of the actual system when the time delay is not taken into account explicitly.

Therefore, a time delay estimation method is needed to successfully apply the approximate realization algorithm to systems with a time delay. In (Helvoirt et al., 2005) the following time delay estimation method is proposed. Suppose that an impulse response sequence $\left\{G_{k}\right\}_{k=0}^{N}$ of length $N+1$ is available and that an appropriate order $\rho$ for the corresponding dynamical system (without the time delay) is known. Supply the sequence $\left\{G_{k+\hat{d}}\right\}_{k=0}^{n}$ with $n+\hat{d}_{\max } \leq N$ to the approximate realization algorithm and determine for which value of $\hat{d}$ the integrated absolute error (IAE) between the original data and the response of the resulting realization, including the added time delay $z^{-\hat{d}}$, is minimal. Note that a reasonable value for the upper bound $\hat{d}_{\max }$ is helpful to minimize computational efforts.

\subsection{Experimental set-up}

Experiments where done with a Varipack 28000 control valve. The experimental set-up is depicted in Fig. 1. The valve is activated through an electro-mechanical actuator that is pneumatically powered and controlled by a 4-20 mA current. The actuator adjusts the position of the valve stem through a set of adjustable levers and beams. It was verified that the static relation between actuator input and valve displacement is linear and that hysteresis in the valve is negligible.

The displacement $y$ of the valve stem was measured by means of a linear variable differential transformator with a range of $2.54 \mathrm{~mm}$, a sensitivity of $0.39 \mathrm{~mm} / \mathrm{V}$, and a cut-off frequency $(-3 \mathrm{~dB})$ of $30 \mathrm{~Hz}$. For data-acquisition a Siglab system with anti-aliasing filters and $\mathrm{AD} / \mathrm{DA}$ converters was used. This system was also used to generate an input signal for the valve. This voltage signal was converted to a current by a galvanic standardsignal isolator with a cut-off frequency $>10 \mathrm{kHz}$.

\section{CONTROL VALVE DYNAMICS}

This section deals with the application of the approximate realization algorithm and time de-

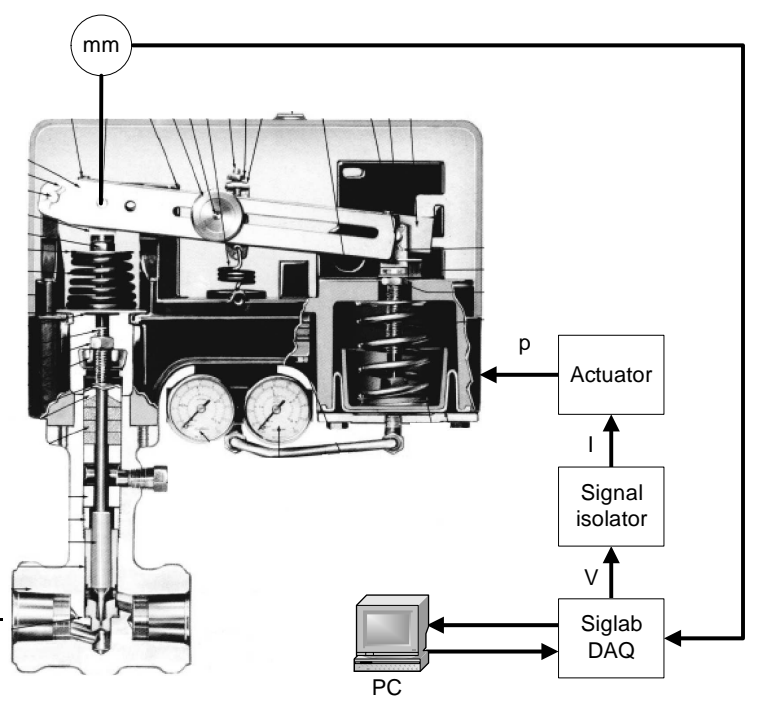

Fig. 1. Experimental set-up.
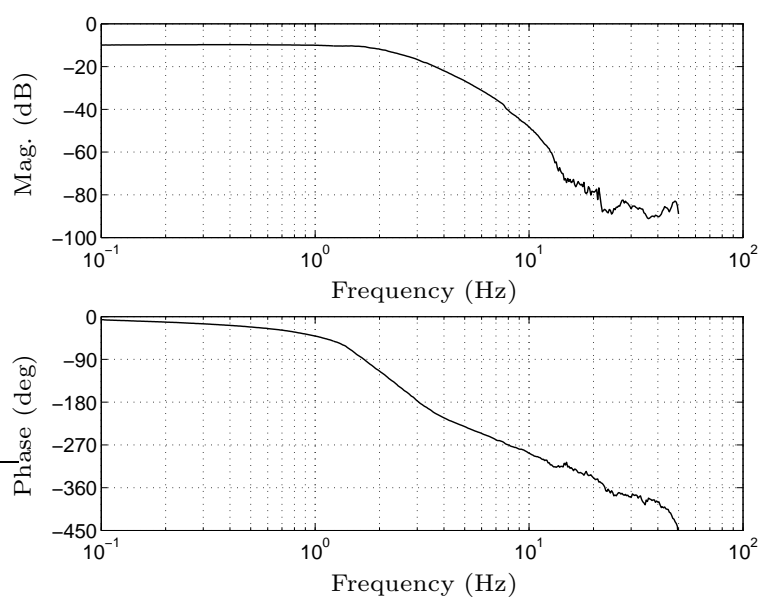

Fig. 2. Measured FRF of valve.

lay estimation method to the control valve under study. First, various measurement results are presented. These results serve both as a reference and a motivation for further analyses. Then the performed time delay estimation and model identification from the measurements are addressed and the obtained results are discussed.

\subsection{Measurements}

With the experimental set-up as described in the previous section, it was possible to measure the frequency response function (FRF) of the control valve. In order to obtain an accurate measurement for the low bandwidth valve (around $2 \mathrm{~Hz}$ ) with sufficient frequency resolution, a stept sine measurement was performed. In total 500 logarithmically spaced points of the FRF were measured in the range of $0.1-50 \mathrm{~Hz}$. For each frequency point the average value of 5 measurements was taken and the accuracy of the input frequency was \pm 0.1 $\mathrm{Hz}$ for each point. The resulting FRF is shown in Fig. 2. 


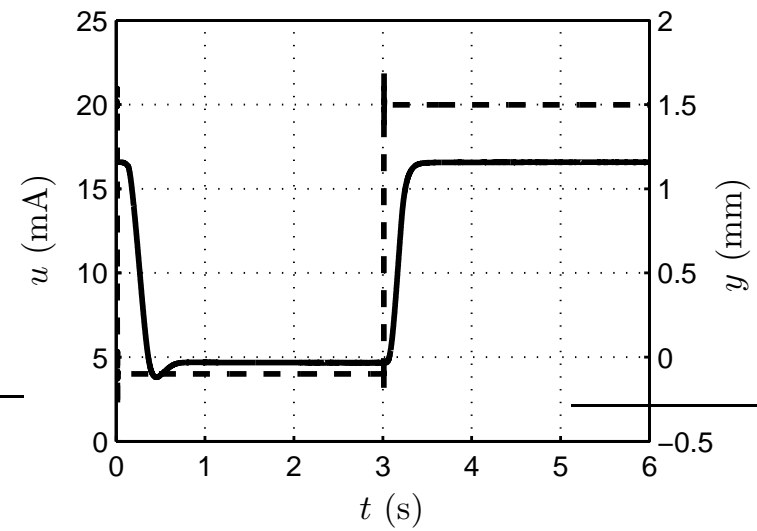

Fig. 3. Step input (- -) and response (-) signals with $A_{s}=16 \mathrm{~mA}$.

It appeared to be difficult to obtain a state space representation that accurately describes the measured FRF with standard frequency domain fitting techniques. Therefore, step response measurements were performed such that the approximate realization algorithm could be applied to obtain a state space model for the valve dynamics. Because the presented algorithm requires an impulse response sequence, numerical differentiation of the measured step response sequences was required. This will be discussed in more detail in the next section.

Step response measurements were carried out for different step sizes $A_{s}$, namely $4,8,12$, and $16 \mathrm{~mA}$, corresponding to $25,50,75$, and $100 \%$ of the full valve range. For each condition 10 measurements (denoted by No. 1 to 10) were done with a sample time $T_{s}$ of $7.8125 \cdot 10^{-4} \mathrm{~s}$ (yielding a Nyquist frequency of $500 \mathrm{~Hz}$ ). The applied step input and the resulting valve response of one particular measurement with $A_{s}=16 \mathrm{~mA}(100 \%)$ are shown in Fig. 3. Note that the actual step is applied just after 3 seconds in order to assure that the system is in a steady-state before the step is applied. The sharp peaks in the input signal are due to imperfections in the driving electronics but their duration is sufficiently short to neglect their influence.

In Fig. 4 a detail of the previous figure is shown that reveals the presence of a time delay in the control valve system. From simulation results it is known that such a time delay will have a negative effect on the achievable accuracy of the approximate realization algorithm. Therefore, an estimate $\hat{d}$ of this time delay is required, such that it can be properly taken into account when the approximate realization algorithm is applied. Estimating the time delay and obtaining a state space model for the valve from step response measurements, will be treated in the next section.

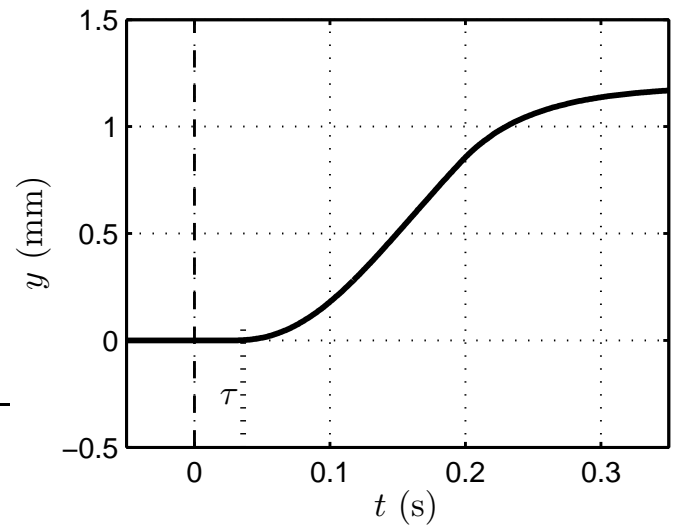

Fig. 4. Delay of approximately $\tau$ seconds in step response signal with $A_{s}=16 \mathrm{~mA}$.

\subsection{Model identification}

As mentioned in the previous section, numerical differentiation of the measured step responses is required to obtain an impulse response sequence to which the approximate realization algorithm can be applied. Differentiation was performed with a third order central difference approximation, yielding errors of $\mathcal{O}\left(T_{s}^{4}\right)$. The resulting impulse response sequences were subsequently filtered through a $5^{\text {th }}$ order, low-pass Butterworth filter with a cut-off frequency of $50 \mathrm{~Hz}$, to remove high frequent noise.

After this pre-processing, the impulse response sequences were then applied to the proposed time delay estimation method. Note that either the impulse response data or the resulting approximate realizations must be properly scaled, since the algorithm is based on the response to a unit impulse function. Because the singular values of the Hankel matrix $H_{E}$ only gave a rough indication for an appropriate order of the approximate realization (see Fig. 5), a suitable value for $\rho$ was determined by trial and error with various values in the range $1-8$, yielding $\rho=3$. Furthermore, the length $n$ of the impulse response sequences was set to 1000 samples and $\hat{d}_{\max }$ was set to 100 .

The resulting IAE as function of the delay $\hat{d}$ is shown in Fig. 6 for one particular measurement (no. 5 with $A_{s}=16 \mathrm{~mA}$ ). The corresponding impulse response of the approximate realization, with the delay value that resulted in a minimal IAE, is shown in Fig. 7, together with the differentiated measurement data.

For other measurements with the same and other step sizes, similar results were obtained. From the $\operatorname{IAE}(\mathrm{d})$-curves the time delay estimate was obtained by determining for which delay value $\hat{d}$ the IAE was minimal. The results for all experiments are summarized in Table 1. By calculating the mean of these results after removing the three 


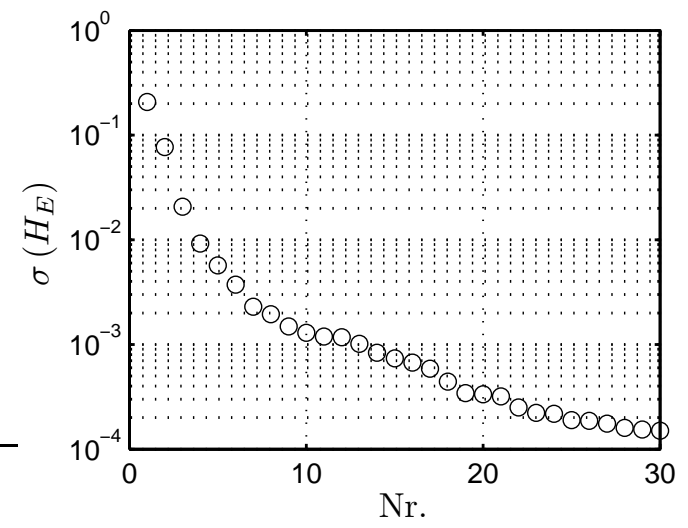

Fig. 5. First 30 singular values of $H_{E}$ for a measurement with $A_{s}=16 \mathrm{~mA}$.

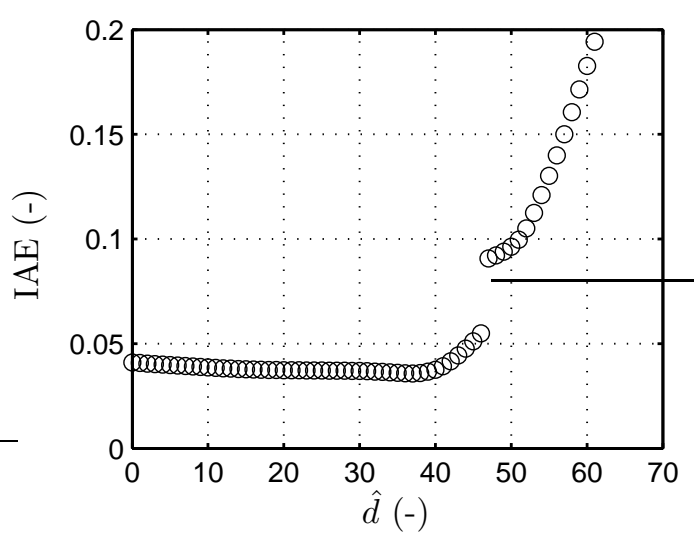

Fig. 6. IAE as function of $\hat{d}$ for a measurement with $A_{s}=16 \mathrm{~mA}$.

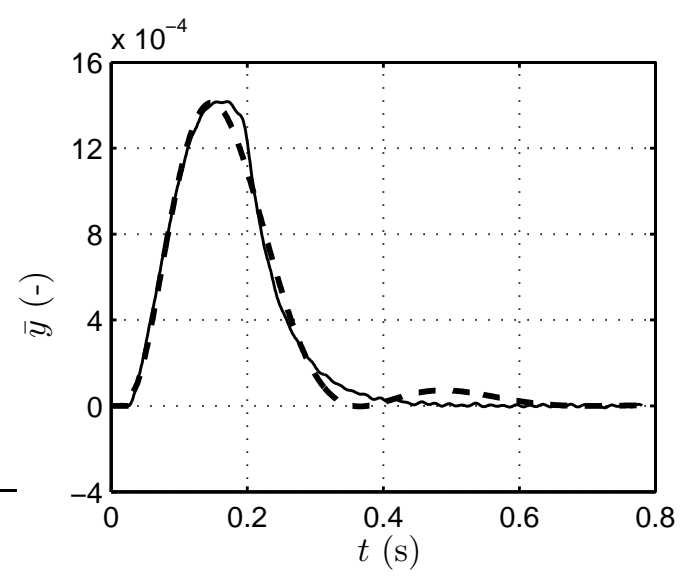

Fig. 7. Normalized impulse responses $\bar{y}$ of realization (- -) with $\hat{d}=37$ and measurement No. 5 (-) with $A_{s}=16 \mathrm{~mA}$.

smallest and highest values, the time delay estimate was determined, yielding $\hat{d}=37$ with a variance $\sigma^{2}=2$. It was confirmed through visual inspection of the raw data that the obtained delay value is accurate.

Finally, in order to obtain a state space representation for the control valve dynamics, the approximate realization algorithm was applied to
Table 1. Estimated time delay values for measurements with different step sizes.

\begin{tabular}{ccccc}
\hline No. & $16 \mathrm{~mA}$ & $12 \mathrm{~mA}$ & $8 \mathrm{~mA}$ & $4 \mathrm{~mA}$ \\
\hline 1 & 38 & 37 & 37 & 37 \\
2 & 37 & 34 & 38 & 38 \\
3 & 38 & 37 & 38 & 38 \\
4 & 36 & 38 & 38 & 37 \\
5 & 37 & 37 & 35 & 38 \\
6 & 34 & 37 & 32 & 38 \\
7 & 34 & 37 & 38 & 38 \\
8 & 33 & 35 & 38 & 38 \\
9 & 36 & 36 & 34 & 35 \\
10 & 38 & 34 & 33 & 38 \\
\hline
\end{tabular}
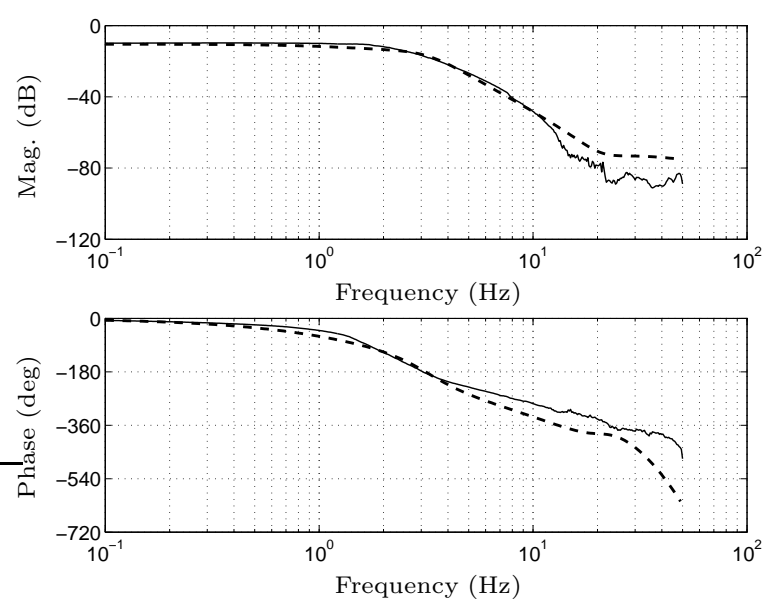

Fig. 8. Measured FRF data (-) and FRF of final approximate realization (- -).

the impulse response sequence that yielded the smallest IAE value for all measurements (no. 2 with $A_{s}=16 \mathrm{~mA}$ ), where the previously determined time delay was taken into account. Together with the measured FRF of the valve, the FRF of the obtained state space model is shown in Fig. 8. Comparing the FRF data shows that the approximate realization algorithm, in combination with the time delay estimation method, provides a reasonable state space model for the valve dynamics in the range of $0.01-13 \mathrm{~Hz}$. It was verified that the frequency of $13 \mathrm{~Hz}$ coincides with the frequency above which the coherence of the FRF measurement starts to drop significantly.

Note however, that there are still quit some differences visible between the two FRFs. Nevertheless, the small variance of the time delay estimate indicates that these differences did not have a significant influence on the time delay estimation.

\section{CONCLUSIONS}

This paper discussed the application of an approximate realization algorithm to obtain a state space realization from impulse response data. In order to deal with the time delay present in the system under study, a time delay estimation method was applied. Both frequency response and step response 
measurements were performed on an experimental set-up for a control valve. The results from the application of the described methods on the experimental data were presented and discussed.

The results showed that the time delay estimation method provided a good estimate for the time delay in the valve system. In addition, by taking the time delay into account, an approximate state space realization could be obtained for the valve dynamics with reasonable accuracy.

Given these encouraging results, it seems worthwhile to generalize the results obtained with the estimation method to other dynamical systems. Furthermore, the robustness and the statistical properties of the time delay estimator should be investigated. Finally, attention should be paid to the influence of measurement noise on the approximate realization algorithm in order to make the algorithm better suitable as an identification tool.

\section{REFERENCES}

Gilbert, E. G. (1963). Controllability and observability in multivariable control systems. SIAM J. on Control 1(2), 128-151.

Helvoirt, J. van, B. de Jager, M. Steinbuch and J. Smeulers (2004). Stability parameter identification for a centrifugal compression system. In: Proc. 43th IEEE Conf. on Decision and Control. Atlantis, Paradise Island, Bahamas. Accepted for publication.

Helvoirt, J. van, O. Bosgra, B. de Jager and M. Steinbuch (2005). Approximate realization with time delay. In: Proc. American Control Conf.. Portland, OR, USA. Submitted for publication.

Ho, B. L. and R. E. Kalman (1965). Effective construction of linear state-variable models from input/output data. In: Proc. 3th Annual Allerton Conf. on Circuit and System Theory. Monticello, IL, USA.

Jang, D., Y. H. Kim and K. S. Hwang (2001). Process identification for second-order plus dead time model using pulse input. In: Proc. 40th SICE Annual Conf.. Nagoya, Japan.

Kalman, R. E. (1963). Mathematical description of linear dynamical systems. SIAM J. on Control 1(2), 152-192.

Kung, S. (1978). A new identification and model reduction algorithm via singular value decompositions. In: Proc. 12th Asilomar Conf. on Circuits, Systems, and Computers. Pacific Grove, CA, USA.

Orlov, Y., L. Belkoura, J. P. Richard and M. Dambrine (2002). On-line parameter identification of linear time-delay systems. In: Proc. 41th IEEE Conf. on Decision and Control. Las Vegas, NV, USA.
Pintelon, R. and L. van Biesen (1990). Identification of transfer functions with time delay and its application to cable fault location. IEEE Trans. Instrum. Meas. 39(3), 479-484.

Schutter, B. de (2000). Minimal state-space realization in linear system theory: An overview. Journal of Computational and Applied Mathematics 121(1-2), 331-354. Special Issue on Numerical Analysis in the 20th CenturyVol. 1: Approximation Theory.

Tuch, J., A. Feuer and Z. J. Palmor (1994). Time delay estimation in continuous linear timeinvariant systems. IEEE Trans. Automat. Contr. 39(4), 823-827. 